\title{
Comparison of different methods for structural analysis of lanthanide-induced NMR shifts: a case of lanthanide(III) cryptates
}

\author{
Carlos F.G.C. Geraldes ${ }^{\mathrm{a}, *}$, Shanrong Zhang ${ }^{\mathrm{b}}$, C. Platas ${ }^{\mathrm{d}}$, T. Rodríguez-Blas ${ }^{\mathrm{d}}$, A. de Blas ${ }^{\mathrm{d}}$, \\ A.D. Sherry ${ }^{\mathrm{b}, \mathrm{c}}$ \\ ${ }^{a}$ Department of Biochemistry, Faculty of Science and Technology, and Center of Neurosciences, University of Coimbra, 3000 Coimbra, Portugal \\ ${ }^{\mathrm{b}}$ Department of Chemistry, The University of Texas at Dallas, P.O. Box 830688, Richardson, TX 75083-0688, USA \\ ${ }^{\mathrm{c}}$ Department of Radiology, The Mary Nell and Ralph B. Rogers Magnetic Resonance Center, University of Texas Southwestern Medical Center, \\ 5801 Forest Park Rd., Dallas, TX 75235-9085, USA \\ ${ }^{\mathrm{d}}$ Departamento de Química Fundamental e Industrial, Universidade da Coruña, Campus de A Zapateira, s/n 15071 A Coruña, Spain
}

\begin{abstract}
The three main methods available in the literature for lanthanide-induced shift (LIS) structural analysis of a series of lanthanide(III) complexes in solution are applied to the data available for a complete series of paramagnetic lanthanide cryptate complexes of a Schiff base axial macrobicyclic ligand $\mathrm{L},[\mathrm{LnL}]^{3+}$. The macrobicycle contracts its cavity as a result of the lanthanide contraction, while preserving the same overall structure in solution. This causes breaks in the LIS plots for all the three methods used. A combined analysis of the data allows to conclude that those breaks reflect abrupt changes of the crystal field parameter $A_{2}^{0}\left\langle r^{2}\right\rangle$ and the hyperfine constants $F_{i}$ of the lanthanides in the middle of the series, but not of the structure of the complex in solution. (C) 2001 Elsevier Science B.V. All rights reserved.
\end{abstract}

Keywords: NMR; Paramagnetic effects; Lanthanide-induced shifts; Solution structures; Cryptate

\section{Introduction}

The binding of a ligand to a paramagnetic $\mathrm{Ln}^{3+}$ ion generally results in large NMR frequency shifts at the ligand nuclei [1], with magnitudes and signs depending critically on both the nature of the $\mathrm{Ln}^{3+}$ ion and the location of the nucleus relative to the metal center. These lanthanide-induced shifts (LIS) are very sensitive to structural changes, allowing many chemical, biophysical and biomedical applications of lanthanide complexes [1-4]. For each nucleus a, the observed shift, $\Delta_{\mathrm{a}}$, is generally a combination of the Fermi contact $\left(\Delta_{\mathrm{c}}\right)$ and dipolar $\left(\Delta_{\mathrm{d}}\right)$ contributions $[5,6]$. For the case of effective axial magnetic symmetry of the complexes, it can be written as:

$\Delta_{\mathrm{a}}=\Delta_{\mathrm{c}}+\Delta_{\mathrm{d}}=\left\langle S_{z}\right\rangle F+D A_{2}^{0}\left\langle r^{2}\right\rangle G$

where $F$ is the hyperfine coupling constant of the ligand nucleus with the $\mathrm{Ln}^{3+}$ ion, $\left\langle S_{z}\right\rangle$ is the spin expectation value [5] for the paramagnetic $\mathrm{Ln}^{3+}, D$ is a magnetic constant proportional to Bleaney's constant $C_{j}$ [6] charac-

*Corresponding author.

E-mail address: geraldes@cygnus.ci.uc.pt (C.F.G.C. Geraldes). teristic of a given $\mathrm{Ln}^{3+}, A_{2}^{0}\left\langle r^{2}\right\rangle$ is a ligand field coefficient of the $\operatorname{Ln}^{3+}$ complex, and $G=\left[\left(3 \cos ^{2} \theta-1\right) / r^{3}\right]$ is a geometric functions of the ligand nucleus, where $(r, \theta, \phi)$ are the polar coordinates of the ligand nucleus in the principal magnetic axis frame of the complex with the $\mathrm{Ln}^{3+}$ ion at the origin.

Because only the dipolar term contains the geometric information about the lanthanide complex, a quantitative structural analysis requires a reliable separation of the observed shift into the contact and dipolar terms. Empirical separation methods have been proposed [1,7], which rely on measurement of LIS data for a group of lanthanide complexes, and are based on a variety of assumptions. The most frequently used graphical separation method [7], is based on rearrangements of Eq. (1) for each nucleus a in two linear forms (Eqs. (2) and (3)):

$$
\begin{aligned}
& \Delta_{\mathrm{a}} /\left\langle S_{z}\right\rangle=F+G A_{2}^{0}\left\langle r^{2}\right\rangle D /\left\langle S_{z}\right\rangle \\
& \Delta_{\mathrm{a}} / D=F\left\langle S_{z}\right\rangle / D+G A_{2}^{0}\left\langle r^{2}\right\rangle
\end{aligned}
$$

The LIS separation is then achieved based on the following assumptions: (1) the hyperfine coupling constants, $F$, and the geometric factor, $G$, are independent of 
the $\mathrm{Ln}^{3+}$ ion; (2) the crystal field parameter, $A_{2}^{0}\left\langle r^{2}\right\rangle$, is invariant along the lanthanide series; (3) the theoretical $\left\langle S_{z}\right\rangle$ and $D$ values available for $\mathrm{Ln}^{3+}$ ions $[5,6]$ are universally valid. Often plots of the observed LIS data according to Eqs. (2) and (3) are linear along the lanthanide series. Then, $F$ and $\left(G A_{2}{ }^{\circ}\left\langle r^{2}\right\rangle\right)$ can be determined by linear regression, and it can be concluded that the various Ln(III) complexes of the ligand studied are isostructural and the crystal field coefficient is invariant along the $\mathrm{Ln}$ (III) series. However, deviations from linearity in those plots, such as breaks or data scatter, often occur [1,2]. Since the ionic radii of the $\mathrm{Ln}^{3+}$ ions decrease across the series from 1.36 to $1.17 \AA$, these deviations may arise from drastic or minor structural changes of the complexes [1]. However, they could also be due to other reasons, such as variation of the crystal field parameter $A_{2}^{0}\left\langle r^{2}\right\rangle[8]$ and/or invalidity of the theoretical $\left\langle S_{z}\right\rangle$ and $D$ values for a specific ligand. Thus, the weakness of this method is that it separately analyses data for one nucleus and depends on two parameters.

In order to better understand the origin of the breaks often observed, following previous work [9], we recently proposed [10] a new method of LIS data analysis following Eq. (4):

$\Delta_{\mathrm{a}} /\left\langle S_{z}\right\rangle=\left(F_{\mathrm{a}}-R_{\mathrm{ab}} F_{\mathrm{b}}\right)+R_{\mathrm{ab}} \Delta_{\mathrm{b}} /\left\langle S_{z}\right\rangle$

which uses LIS data for two given nuclei a and $b$. Plots of $\Delta_{\mathrm{a}} /\left\langle S_{z}\right\rangle$ versus $\Delta_{\mathrm{b}} /\left\langle S_{z}\right\rangle$ give, through their slope, the ratio of the $G$ values of nuclei a and b, $R_{\mathrm{ab}}=G_{\mathrm{a}} / G_{\mathrm{b}}$, and the intercept, the value of $\left(F_{\mathrm{a}}-R_{\mathrm{ab}} F_{\mathrm{b}}\right)$. For isostructural complexes, these plots are linear if $F_{i}$ are constant or any breaks present divide the data in parallel lines if $F_{i}$ change along the $\mathrm{Ln}(\mathrm{III})$ series. The advantage of this procedure is that it does not require the assumption of constancy of the ligand field coefficient $A_{2}^{0}\left\langle r^{2}\right\rangle$ along the $\operatorname{Ln}(\mathrm{III})$ series, as it depends only on the hyperfine coupling constants.

We also recently proposed [11] a new method to check the isostructurality for a series of $\mathrm{Ln}^{3+}$ complexes, which is independent of the theoretical $\left\langle S_{z}\right\rangle$ and $D$ values, as well as the crystal field parameter. It is solely based on the experimental LIS data for three nuclei a, b and c, $\Delta_{\mathrm{a}}, \Delta_{\mathrm{b}}$ and $\Delta_{c}$, through Eq. (5) [11]:

$\Delta_{\mathrm{a}} / \Delta_{\mathrm{b}}=\alpha \Delta_{\mathrm{c}} / \Delta_{\mathrm{b}}+\beta$

where $\quad \alpha=\left(S_{\mathrm{ab}}-R_{\mathrm{ab}}\right) /\left(S_{\mathrm{cb}}-R_{\mathrm{cb}}\right) \quad$ and $\quad \beta=\left(S_{\mathrm{cb}} R_{\mathrm{ab}}-\right.$ $\left.S_{\mathrm{ab}} R_{\mathrm{cb}}\right) /\left(S_{\mathrm{cb}}-R_{\mathrm{cb}}\right) \quad$ in which $S_{\mathrm{ab}}=F_{\mathrm{a}} / F_{\mathrm{b},} \quad S_{\mathrm{cb}}=F_{\mathrm{c}} / F_{\mathrm{b}}$, $R_{\mathrm{ab}}=G_{\mathrm{a}} / G_{\mathrm{b}}$ and $R_{\mathrm{cb}}=G_{\mathrm{c}} / G_{\mathrm{b}}$. Plotting $\Delta_{\mathrm{a}} / \Delta_{\mathrm{b}}$ against $\Delta_{\mathrm{c}} /$ $\Delta_{\mathrm{b}}$ should yield a straight line with a slope of $\alpha$ and intercept of $\beta$, provided that $S_{\mathrm{ab}}$ and $S_{\mathrm{cb}}$, and $R_{\mathrm{ab}}$ and $R_{\mathrm{cb}}$ are invariant along the lanthanide series. The simplicity of Eq. (5) makes it very easy to analyse the isostructurality of a well-defined series of lanthanide complexes [11]. It utilizes exclusively the experimental shift data to test for the invariance of the structure-dependent parameters $F$ and $G$ ratios along the lanthanide(III) series, without recourse
(5)

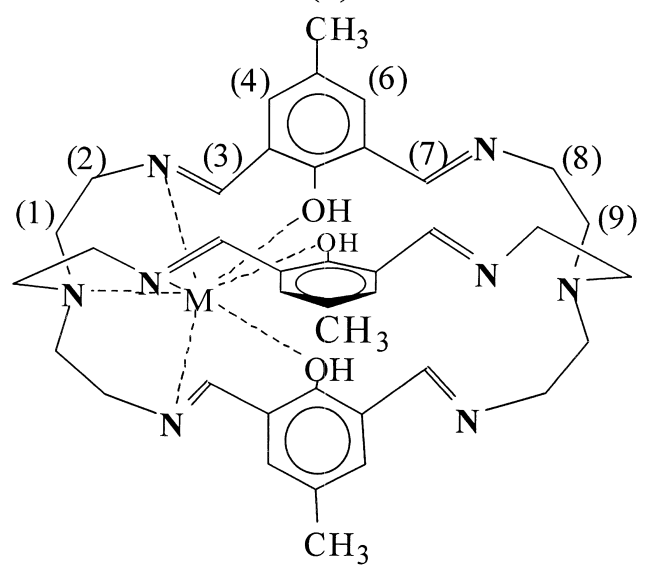

Fig. 1. Schematic structure and proton numbering of the lanthanide cryptate complexes of the Schiff base axial macrobicyclic ligand L, $[\mathrm{LnL}]^{3+}$.

to the theoretical $\left\langle S_{z}\right\rangle$ and $D$ values, and is also independent of the crystal field parameter $A_{2}^{0}\left\langle r^{2}\right\rangle$.

In this work, these three main methods are applied to analyse LIS data available [10] for a complete series of paramagnetic lanthanide cryptate complexes of a Schiff base axial macrobicyclic ligand $\mathrm{L},[\mathrm{LnL}]^{3+}(\mathrm{Ln} \neq \mathrm{La}, \mathrm{Gd}$, Y, Lu) (see Fig. 1). By comparison with the available crystal structures, the applicability of these three methods is critically evaluated.

\section{Results and discussion}

The LIS data available for the protons of the complete series of paramagnetic lanthanide cryptate complexes of the Schiff base axial macrobicyclic ligand L [10] were plotted according to Eqs. (2) and (3). Many of the plots of $\Delta_{\mathrm{a}} /\left\langle S_{z}\right\rangle$ versus $D /\left\langle S_{z}\right\rangle$ and of $\Delta_{\mathrm{a}} / D$ versus $\left\langle S_{z}\right\rangle / D$ do not follow a single linear correlation but rather divide into two subgroups $(\mathrm{Ln}=\mathrm{Ce}-\mathrm{Eu}$ and $\mathrm{Ln}=\mathrm{Tb}-\mathrm{Yb}$ ) with a break located near the middle of the lanthanide series, as illustrated in Fig. 2 for the $\mathrm{H}_{3}$ protons. These plots for the other nuclei also show breaks, more evident for some nuclei than for others. The values of $A_{2}^{0}\left\langle r^{2}\right\rangle G$ and $F$ were obtained for all protons [10]. Their values (Table 1) change from the first to the second half of the $\operatorname{Ln}(\mathrm{III})$ series.

The same sets of LIS data for protons of these lanthanide cryptate complexes were also plotted according to Eq. (4). These plots originate two straight lines for the light and heavy lanthanides, respectively, which are almost parallel as typically shown in Fig. 3 for the $\mathrm{H}_{1}$ and $\mathrm{H}_{3}$ protons. The values of $R_{\mathrm{ab}}$ and of $\left(F_{\mathrm{a}}-R_{\mathrm{ab}} F_{\mathrm{b}}\right)$, obtained by fitting the experimental LIS data according to Eq. (4), are in good agreement with those calculated from the values of $A_{2}^{0}\left\langle r^{2}\right\rangle G$ and $F$ using the previous method (Table 1). The plots and the fitted parameters clearly indicate that there is 


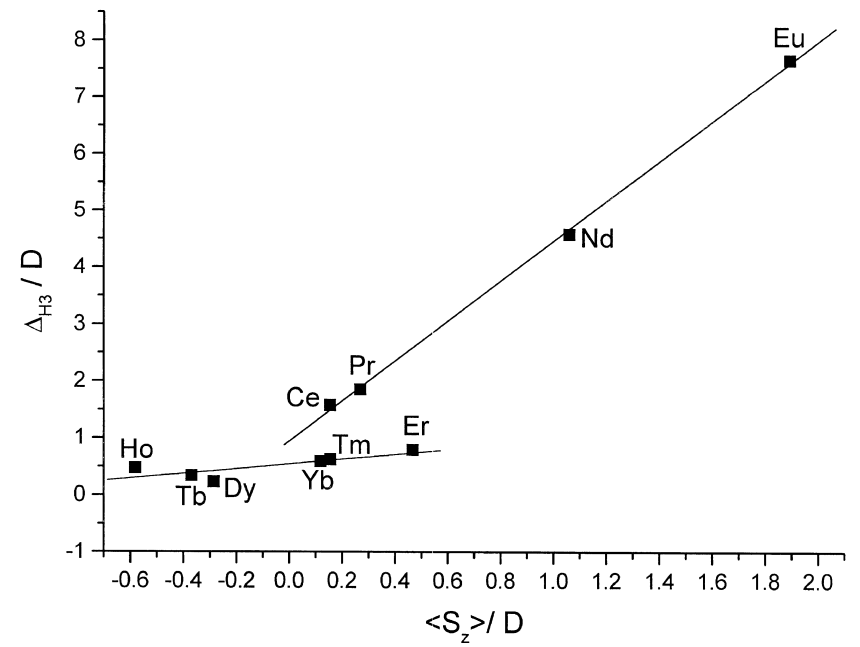

Fig. 2. Plot for separation of contact and pseudocontact contributions to the proton LIS data ( $\mathrm{H}_{3}$ protons) according to Eq. (3).

no drastic change in the ratios of the geometric terms, $R_{\mathrm{ab}}$, along the lanthanide series, although the geometric terms themselves vary. The $A_{2}^{0}\left\langle r^{2}\right\rangle G$ values of the first and second half of the lanthanide series differ only by a

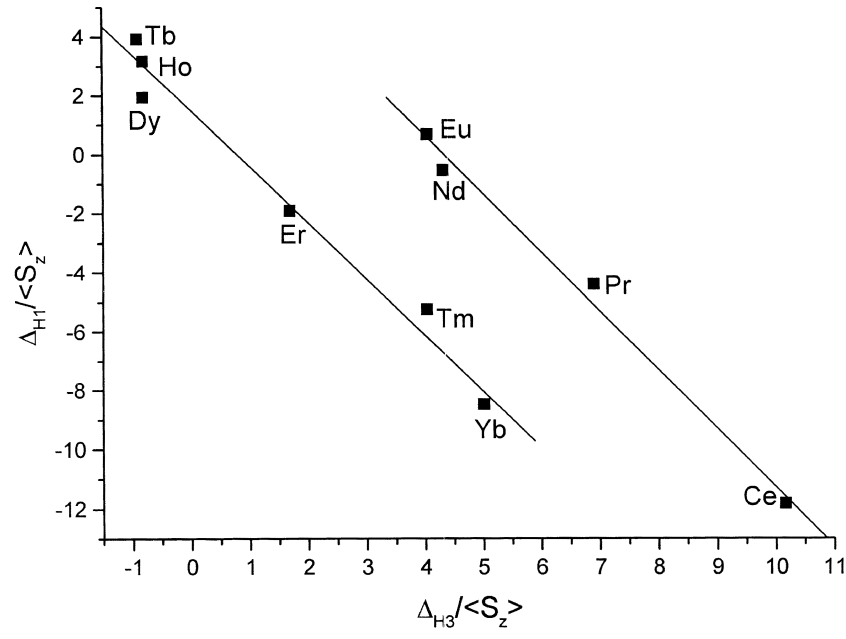

Fig. 3. Plot of the proton LIS values $\left(\mathrm{H}_{1}\right.$ and $\left.\mathrm{H}_{3}\right)$ according to Eq. (4). The average LIS values of $\mathrm{H}_{1 \mathrm{ax}}$ and $\mathrm{H}_{1 \mathrm{eq}}$ were used.

proportionality constant, but the $R_{\mathrm{ab}}$ ratios are constant along the whole series. The very different $\left(F_{\mathrm{a}}-R_{\mathrm{ab}} F_{\mathrm{b}}\right)$ values obtained reflect a drastic change in the proton hyperfine constants along the lanthanide series. Thus, the

Table 1

Comparison of parameters depending on geometric ratios and hyperfine coupling constants obtained from plots of the proton LIS data for the $[\mathrm{Ln}(\mathrm{L})]^{3+}$ complexes according to Eqs. (2)-(5)

\begin{tabular}{|c|c|c|c|c|}
\hline \multirow[t]{2}{*}{ Nuclei $^{a}$} & \multicolumn{2}{|l|}{$\mathrm{Ce} \rightarrow \mathrm{Eu}$} & \multicolumn{2}{|l|}{$\mathrm{Tb} \rightarrow \mathrm{Yb}$} \\
\hline & $\overline{F_{\mathrm{a}}}$ & $A_{2}^{0}\left\langle r^{2}\right\rangle G_{\mathrm{a}}$ & $\overline{F_{\mathrm{a}}}$ & $A_{2}^{0}\left\langle r^{2}\right\rangle G_{\mathrm{a}}$ \\
\hline $\mathrm{H}_{1 \mathrm{ax}}$ & $1.81 \pm 0.28$ & $-2.05 \pm 0.22$ & $0.33 \pm 0.32$ & $-1.06 \pm 0.09$ \\
\hline $\mathrm{H}_{1 \mathrm{eq}}$ & $1.30 \pm 0.25$ & $-1.78 \pm 0.15$ & $0.42 \pm 0.34$ & $-0.95 \pm 0.11$ \\
\hline $\mathrm{H}_{2 \mathrm{ax}}$ & $0.55 \pm 0.13$ & $-0.06 \pm 0.04$ & $0.79 \pm 0.13$ & $-0.09 \pm 0.05$ \\
\hline $\mathrm{H}_{2 \mathrm{eq}}$ & $0.40 \pm 0.10$ & $-0.01 \pm 0.04$ & $0.33 \pm 0.05$ & $-0.05 \pm 0.02$ \\
\hline $\mathrm{H}_{3}$ & $3.48 \pm 0.06$ & $1.03 \pm 0.05$ & $0.57 \pm 0.17$ & $0.52 \pm 0.05$ \\
\hline $\mathrm{H}_{4}$ & $0.25 \pm 0.04$ & $0.49 \pm 0.04$ & $0.40 \pm 0.15$ & $0.55 \pm 0.05$ \\
\hline \multirow[t]{2}{*}{$\mathrm{H}_{5}^{4}$} & $0.01 \pm 0.02$ & $0.18 \pm 0.01$ & $-0.11 \pm 0.01$ & $0.06 \pm 0.01$ \\
\hline & $R_{\mathrm{ab}}$ & $\left(F_{\mathrm{a}}-R_{\mathrm{ab}} F_{\mathrm{b}}\right)$ & $R_{\mathrm{ab}}$ & $\left(F_{\mathrm{a}}-R_{\mathrm{ab}} F_{\mathrm{b}}\right)$ \\
\hline \multirow{2}{*}{$\underset{\text { calc }^{\mathrm{c}}}{a=\mathrm{H}_{5}, b}=\mathrm{H}_{3} \exp ^{\mathrm{b}}$} & $0.177 \pm 0.009$ & $-0.58 \pm 0.06$ & $0.122 \pm 0.009$ & $-0.16 \pm 0.02$ \\
\hline & 0.175 & -0.60 & 0.115 & -0.17 \\
\hline \multirow{2}{*}{$\begin{array}{l}a=\mathrm{H}_{1}, b=\mathrm{H}_{3} \exp ^{\mathrm{b}} \\
\text { calc }^{\mathrm{c}}\end{array}$} & $-2.00 \pm 0.11$ & $8.71 \pm 0.92$ & $-1.73 \pm 0.22$ & $1.49 \pm 0.40$ \\
\hline & -1.86 & 8.03 & -1.93 & 1.48 \\
\hline \multirow{3}{*}{$\begin{array}{l}a=\mathrm{H}_{4}, b=\mathrm{H}_{3} \exp ^{\mathrm{b}} \\
\text { calc }^{\mathrm{c}}\end{array}$} & $0.53 \pm 0.02$ & $-1.64 \pm 0.12$ & $1.03 \pm 0.08$ & $0.03 \pm 0.03$ \\
\hline & 0.48 & -1.42 & 1.06 & -0.03 \\
\hline & $\alpha$ & $\beta$ & $\alpha$ & $\beta$ \\
\hline \multirow{2}{*}{$\begin{array}{c}a=\mathrm{H}_{\mathrm{leq}}, b=\mathrm{H}_{3} c=\mathrm{H}_{5} \exp ^{\mathrm{d}} \\
\text { calc }^{\mathrm{e}}\end{array}$} & -12.21 & 0.41 & -8.32 & -0.31 \\
\hline & -12.98 & 0.44 & -9.76 & -0.31 \\
\hline \multirow{2}{*}{ 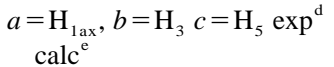 } & -14.59 & 0.60 & -8.49 & 1.06 \\
\hline & -14.03 & 0.59 & -13.8 & 0.31 \\
\hline \multirow{2}{*}{$\begin{array}{l}a=\mathrm{H}_{2 \mathrm{eq}}, b=\mathrm{H}_{3} c=\mathrm{H}_{5} \exp ^{\mathrm{d}} \\
\operatorname{calc}^{\mathrm{e}}\end{array}$} & -0.73 & 0.12 & -2.19 & 0.16 \\
\hline & -0.83 & 0.11 & -1.46 & 0.13 \\
\hline \multirow{2}{*}{ 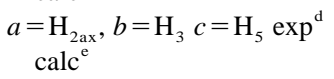 } & -1.26 & 0.16 & -5.06 & 0.41 \\
\hline & -1.00 & 0.16 & -5.18 & 0.44 \\
\hline \multirow{2}{*}{$\begin{array}{c}a=\mathrm{H}_{4}, b=\mathrm{H}_{3} c=\mathrm{H}_{5} \exp ^{\mathrm{d}} \\
\operatorname{calc}^{\mathrm{e}}\end{array}$} & 2.35 & 0.06 & 1.10 & 0.93 \\
\hline & 2.60 & 0.06 & 0.16 & 0.93 \\
\hline
\end{tabular}

${ }^{a}$ Parameters obtained from plots of the proton LIS data according to Eqs. (2) and (3).

${ }^{\mathrm{b}}$ Parameters obtained from plots according to Eq. (4).

${ }^{c}$ Parameters calculated from data of Footnote a.

${ }^{\mathrm{d}}$ Parameters obtained from plots according to Eq. (5).

e Parameters calculated from data of Footnote a. 
breaks in the plots, according to Eqs. (3) and (4), found in the middle of the $\mathrm{Ln}$ series, are due to a variation of the crystal field parameter $A_{2}^{0}\left\langle r^{2}\right\rangle[8]$ and the hyperfine constants $F_{i}$ in the middle of the Ln series.

Finally, the same LIS data were plotted according to Eq. (5). These plots also show a break from the light to the heavy lanthanides, as illustrated in Fig. 4 for protons $\mathrm{H}_{2 \mathrm{ax}}$, $\mathrm{H}_{3}$ and $\mathrm{H}_{5}$. The values of the $\alpha$ and $\beta$ parameters, obtained by fitting the experimental LIS data to Eq. (5), are generally in fairly good agreement with those calculated from the values of $A_{2}^{0}\left\langle r^{2}\right\rangle G$ and $F$ using the first method (Table 1). Those parameters, which depend on the $R_{i k}$ geometric ratios and the $S_{i k}$ ratios of hyperfine constants $F_{i}$ and $F_{k}$, change quite significantly from the first to the second half of the lanthanide series. This change is thus due to the change of the hyperfine constants but not the geometric terms of the complexes.

X-ray crystal structures and solution NMR structural studies have shown that the entire series of these lanthanide cryptate complexes all adopt very similar structures. Lanthanide contraction only leads to a slight distortion of the coordination polyhedron and contraction of the macrobicycle cavity but no drastic structural difference is seen between the light $(\mathrm{Ce}-\mathrm{Eu})$ and heavy $(\mathrm{Tb}-\mathrm{Yb})$ complexes [10]. This gradual decrease of the Ln-donor atom bond distances as one progresses along the series, causes a variation of the crystal field parameter $A_{2}^{0}\left\langle r^{2}\right\rangle$ and the hyperfine constants $F_{i}$ in the middle of the series. However, it affects only slightly on the geometric terms $G_{i}$ of the protons. These kinds of minor geometric variations are

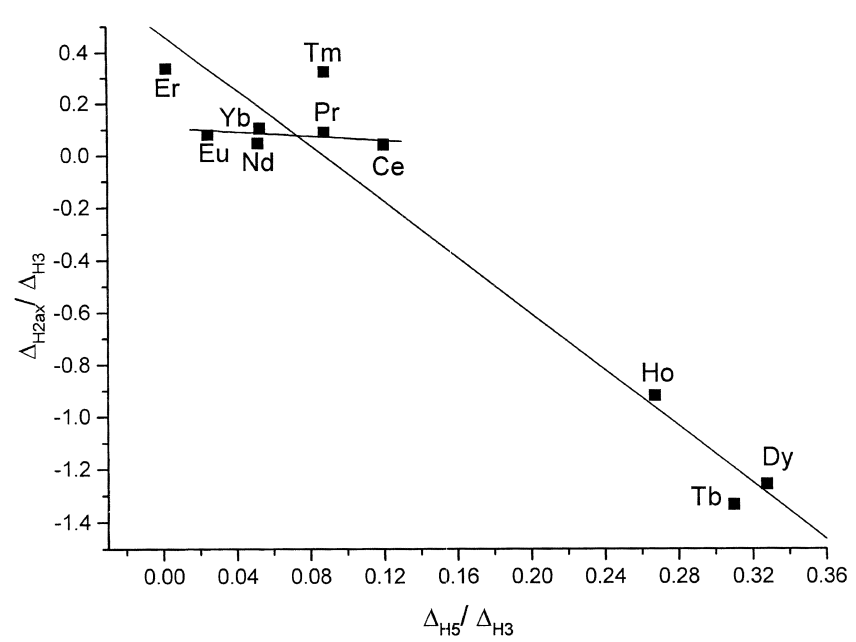

Fig. 4. Plot of the proton LIS values $\left(\mathrm{H}_{2 \mathrm{ax}}, \mathrm{H}_{3}\right.$ and $\left.\mathrm{H}_{5}\right)$ according to Eq. (5). nearly invisible in the geometric ratios of $R_{i k}$. Considering the above facts, one can understand the real reasons of observing the breaks in the different plots according to all the three methods used. In the first method, which uses only the LIS from one nucleus in a plot and depends on the three $\operatorname{Ln}\left(\right.$ III) parameters $A_{2}^{0}\left\langle r^{2}\right\rangle, D$ and $\left\langle S_{z}\right\rangle$, the breaks originate from both changes of $A_{2}^{0}\left\langle r^{2}\right\rangle$ and $F_{i}$. In the second method, which uses the LIS from two nuclei and depends on one $\operatorname{Ln}\left(\right.$ III) parameter $\left\langle S_{z}\right\rangle$, the breaks result from changes of $F_{i}$. Finally, in the third method, which is independent of all the $\operatorname{Ln}\left(\right.$ III) parameters $A_{2}^{0}\left\langle r^{2}\right\rangle, D$ and $\left\langle S_{z}\right\rangle$, the observed breaks also result from changes of $F_{i}$, which contribute to the $\alpha$ and $\beta$ parameters. Therefore, this parameter-independent method, when used by itself as a test of isostructurality, cannot distinguish between changes of geometric factors and changes of $F_{i}$ values, as the second method does. Thus, we advocate the simultaneous use of these three methods in a structural analysis.

\section{Acknowledgements}

Support of this research by grants from Fundação da Ciência e Tecnologia, Portugal (PRAXIS 2/2.1/SAU/ 1194/96), the Robert A. Welch Foundation (AT-584), the NIH Biotechnology Research Program (RR02584) and Xunta de Galicia (XUGA PGID99PXI20902B) is gratefully acknowledged. This work was carried out in the framework of COST D18 Action of the E.U.

\section{References}

[1] J.A. Peters, J. Huskens, D.J. Raber, Prog. NMR Spectrosc. 28 (1996) 283.

[2] A.D. Sherry, C.F.G.C. Geraldes, in: J.-C.G. Bünzli, G.R. Choppin (Eds.), Lanthanide Probes in Life, Chemical and Earth Sciences, Elsevier, Amsterdam, 1989.

[3] M.C. Alpoim, A.M. Urbano, C.F.G.C. Geraldes, J.A. Peters, J. Chem. Soc. Dalton Trans. (1992) 463.

[4] D.C. Buster, M.M.C.A. Castro, C.F.G.C. Geraldes, C.R. Malloy, A.D. Sherry, T.C. Siemers, Magn. Reson. Med. 15 (1990) 25.

[5] R.M. Goding, M.P. Halton, Aust. J. Chem. 25 (1972) 2577.

[6] B. Bleaney, J. Magn. Reson. 8 (1972) 91.

[7] C.N. Reilley, B.W. Good, R.D. Allendoerfer, Anal. Chem. 48 (1976) 1446.

[8] J. Ren, A.D. Sherry, J. Magn. Reson. B111 (1996) 178.

[9] C. Platas, F. Avecilla, A. de Blas, C.F.G.C. Geraldes, T. RodriguezBlas, H. Adams, J. Mahia, Inorg. Chem. 31 (1999) 3190.

[10] J. Reuben, J. Magn. Reson. 50 (1982) 233.

[11] R. Ren, S. Zhang, C.F.G.C. Geraldes, A.D. Sherry (submitted). 